\title{
ANÁLISE DINÂMICA NÃO LINEAR DE PISOS MISTOS DE EDIFICAÇÕES SUB- METIDOS A ATIVIDADES HUMANAS RÍTMICAS
}

\author{
E. D. C. Lopes ${ }^{1}$, J.G.S. Silva ${ }^{2}$, S. A. L. Andrade ${ }^{1}$ \\ ${ }^{1}$ Departamento de Engenharia Civil, Pontifícia Universidade Católica do Rio de Janeiro, \\ PUC-Rio
}

${ }^{2}$ Departamento de Estruturas e Fundações, Universidade do Estado do Rio de Janeiro, UERJ (jgss@uerj.br)

Resumo. A crescente incidência de problemas de vibração em edifícios, devido às atividades humanas, levou a um critério específico a ser abordado nos projetos estruturais. Esta foi a principal motivação para o desenvolvimento de uma metodologia de projeto centrado nos pisos mistos, de resposta dinâmica não linear, submetido a ações dinâmicas humanas. $O$ objetivo principal deste trabalho é investigar a influência do grau de interação aço-concreto (do total a vários níveis de interação parcial). O sistema estrutural foi composto por uma solução mista (aço-concreto), feito de um perfil de aço "I" e uma laje de concreto armado. A metodologia de análise proposta adotou as técnicas usuais de discretização, via método dos elementos finitos (MEF), por meio do emprego do programa Ansys. Um estudo paramétrico foi desenvolvido com foco na determinação da influência da interação de aço-concreto sobre a resposta dinâmica do piso misto. As acelerações de pico encontradas foram comparadas com os valores limites propostas por vários autores e padrões de concepção. Os resultados numéricos indicam que a modelagem das excitações dinâmicas foi um dos principais pontos chave para obtenção de resultados confiáveis. A presente investigação indica que atividades humanas rítmicas podem induzir os pisos mistos a alcançar níveis de vibração inaceitáveis e, nestas situações, conduzir a uma violação dos atuais critérios de conforto humano para essas estruturas específicas.

Palavras-chave: Análises dinâmicas não lineares, pisos mistos, atividades humanas rítmicas.

\section{INTRODUÇÃO}

Os sistemas estruturais compostos por pisos mistos (aço-concreto) têm sido bastante utilizados em obras de engenharia civil, atendendo as necessidades impostas pelo arrojo da arquitetura moderna, onde existe uma necessidade premente de se vencer grandes vãos, utilizando elementos estruturais com as menores dimensões possíveis. Diante deste cenário, esse tipo de sistema estrutural, pisos mistos (aço-concreto), tem apresentado problemas crescentes associados a vibrações excessivas. 
Assim sendo, recomenda-se que o conhecimento acerca do comportamento estrutural destes pisos mistos (aço-concreto), seja cuidadosamente investigado, quando da atuação de carregamentos dinâmicos, especialmente aqueles nocivos ao conforto humano dos usuários e, ainda, às partes constituintes do sistema estrutural. Para tanto, nos últimos anos, diversos estudos nesta área de pesquisa vem sendo realizados sob os mais variados enfoques por diversos pesquisadores [1-7].

De acordo com este contexto, este trabalho de pesquisa objetiva contribuir com o desenvolvimento de uma metodologia de análise para estudo do comportamento dinâmico de modelos estruturais associados a pisos mistos (aço-concreto), submetidos a ações dinâmicas humanas rítmicas, nos quais as ligações estruturais viga-coluna e viga-viga sejam do tipo rígidas, semirrígidas e flexíveis. Objetiva-se, ainda, desenvolver um estudo sobre os níveis de interação entre os materiais aço e concreto, considerando-se os efeitos provenientes da interação total e parcial entre a laje de concreto e as vigas de aço. A não linearidade geométrica, presente nestes sistemas de pisos também será alvo de estudo.

A definição das ações dinâmicas atuantes sobre os modelos estruturais foi feita com base em resultados experimentais, os quais levam em conta os efeitos de multidão. A análise fundamenta-se na modelagem computacional dos sistemas estruturais, através do Método dos Elementos Finitos (MEF). São empregadas técnicas usuais de discretização, por meio do programa Ansys [8].

A resposta dinâmica dos modelos contempla uma análise crítica acerca dos valores das acelerações máximas (acelerações de pico). Os resultados obtidos, em termos das acelerações de pico são confrontados e comparados com os limites propostos por recomendações internacionais, sob o ponto de vista do conforto humano, para diferentes tipos de ocupação.

\section{CRITÉRIOS DE CONFORTO HUMANO}

Sabe-se que ao projetar um piso misto (aço-concreto) em que as suas frequências naturais, especialmente a frequência fundamental, estejam próximas das frequências da excitação mais comuns, tais como aquelas referentes às atividades humanas do caminhar, pular, saltar e dançar, certamente o sistema apresentará problemas referentes a vibrações excessivas.

As cargas dinâmicas podem produzir elevados níveis de vibração, que podem tanto comprometer a segurança estrutural como causar alteração na sensação de conforto dos ocupantes dessas edificações. Por outro lado essas atividades físicas têm sido consideradas na análise dinâmica de pisos de edificações de forma cada vez mais elaborada, pois a modelagem deste tipo de carregamento dinâmico é bastante complexa, devido às particularidades corporais de cada indivíduo e, bem como, a maneira que realizam um determinado tipo de ação [3].

Nesta investigação estuda-se a resposta dinâmica de pisos mistos quando submetidos a essas ações dinâmicas humanas. Um modelo de carregamento foi idealizado, objetivando a representação da ação dinâmica gerada por atividades humanas (ginástica aeróbica e saltos à vontade) sobre pisos de edificações. Na sequência do texto, o modelo de carregamento empregado ao longo da presente investigação será descrito mais detalhadamente. 


\subsection{Modelo de carregamento}

O modelo de carregamento empregado neste trabalho de pesquisa foi desenvolvido para simular as excitações dinâmicas humanas rítmicas como a ginástica aeróbica e saltos à vontade e a função Hanning é empregada para descrever matematicamente este carregamento [2]. A representação matemática deste tipo de carregamento dinâmico é feita por meio da função Hanning, dada pelas Equações (1) e (2).

$$
\begin{gathered}
F(t)=C D\left\{K_{p} P\left[0,5-0,5 \cos \left(2 \pi t / T_{C}\right)\right]\right\} \cdot \text { para } t \leq T \\
F(t)=0 \cdot \text { para } T_{c}<t \leq T
\end{gathered}
$$

Onde:

$\mathrm{F}(\mathrm{t})$ = representação matemática do carregamento no tempo em $(\mathrm{N})$;

$\mathrm{CD}=$ coeficiente de defasagem;

$\mathrm{K}_{\mathrm{p}} \quad=$ coeficiente de impacto;

$\mathrm{P} \quad=$ peso da pessoa em $(\mathrm{N})$;

$\mathrm{T} \quad=$ período da atividade em (s);

$\mathrm{T}_{\mathrm{c}} \quad=$ período de contato da atividade em (s);

$\mathrm{T}=$ tempo em (s).

A Figura 1 facilita o entendimento da função, pois esta ilustra os dois intervalos de tempo significativos da referida função. Ressalta-se que a força dinâmica normalizada (FDN) é representada na ordenada da Figura 1.

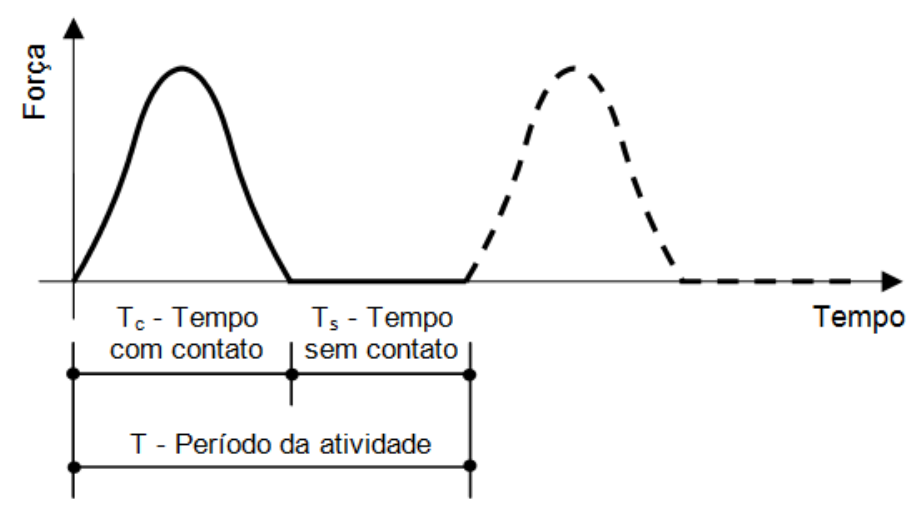

Figura 1. Representação genérica da função de carregamento dinâmico.

Desses parâmetros, citados anteriormente, destaca-se o coeficiente de impacto $\mathrm{K}_{\mathrm{p}}$, é um coeficiente de majoração da carga, que considera o impacto do salto sobre a estrutura. $\mathrm{O}$ coeficiente de defasagem CD é um coeficiente de ponderação das ações dinâmicas definidas em função da atividade realizada e do número de pessoas que atuam sobre a estrutura. Este coeficiente leva em conta os efeitos de multidão, ou seja, o grau de sincronismo entre as pessoas que atuam sobre o piso [2]. Deste modo, a definição deste parâmetro considera possíveis defasagens e variações de ritmo que levariam a redução da intensidade do carregamento. $\mathrm{Na}$ sequência, são apresentados os valores do coeficiente de defasagem, CD, e os demais parâmetros obtidos experimentalmente [2], para três tipos de atividades humanas rítmicas, Tabela 1. 
Tabela 1. Valores adotados para o coeficiente de defasagem CD [2]

\begin{tabular}{ccc}
\hline \multirow{2}{*}{ Número de Pessoas } & \multicolumn{2}{c}{ CD } \\
\cline { 2 - 3 } & Ginástica Aeróbica & Saltos à Vontade \\
\hline 1 & 1 & 1 \\
3 & 1 & 0,88 \\
6 & 0,97 & 0,74 \\
9 & 0,96 & 0,70 \\
12 & 0,95 & 0,67 \\
16 & 0,94 & 0,64 \\
24 & 0,93 & 0,62 \\
32 & 0,92 & 0,60 \\
\hline
\end{tabular}

Tabela 2. Parâmetros utilizados na função de carregamento [2]

\begin{tabular}{cccc}
\hline Atividades & $\mathrm{T}(\mathrm{s})$ & $\mathrm{T}_{\mathrm{c}}(\mathrm{s})$ & $\mathrm{K}_{\mathrm{p}}$ \\
\hline Saltos à vontade & $0,44 \pm 0,15$ & $0,32 \pm 0,09$ & $3,17 \pm 0,58$ \\
Ginástica aeróbica & $0,44 \pm 0,09$ & $0,34 \pm 0,09$ & $2,78 \pm 0,60$ \\
Show/Torcida & $0,37 \pm 0,03$ & $0,37 \pm 0,03$ & $2,41 \pm 0,51$ \\
\hline
\end{tabular}

A Figura 2 apresenta exemplos de sinais da força no tempo correspondente à ginástica aeróbica. Para tal, foram utilizados os seguintes parâmetros: $T_{c}=0,34 s, T_{s}=0,44 s, K_{p}=2,78$ e o coeficiente $\mathrm{CD}=0,92$. O peso de um ser humano foi considerado como sendo igual a $800 \mathrm{~N}$ [9]. O amortecimento estrutural foi considerado como sendo igual a $1 \%(\xi=1 \%)$ [6]. Os carregamentos dinâmicos aplicados sobre os modelos correspondem a trinta e duas pessoas, Figura 7. A distribuição foi feita utilizando uma taxa de ocupação de 0,25 pessoas $/ \mathrm{m}^{2}$ [9].
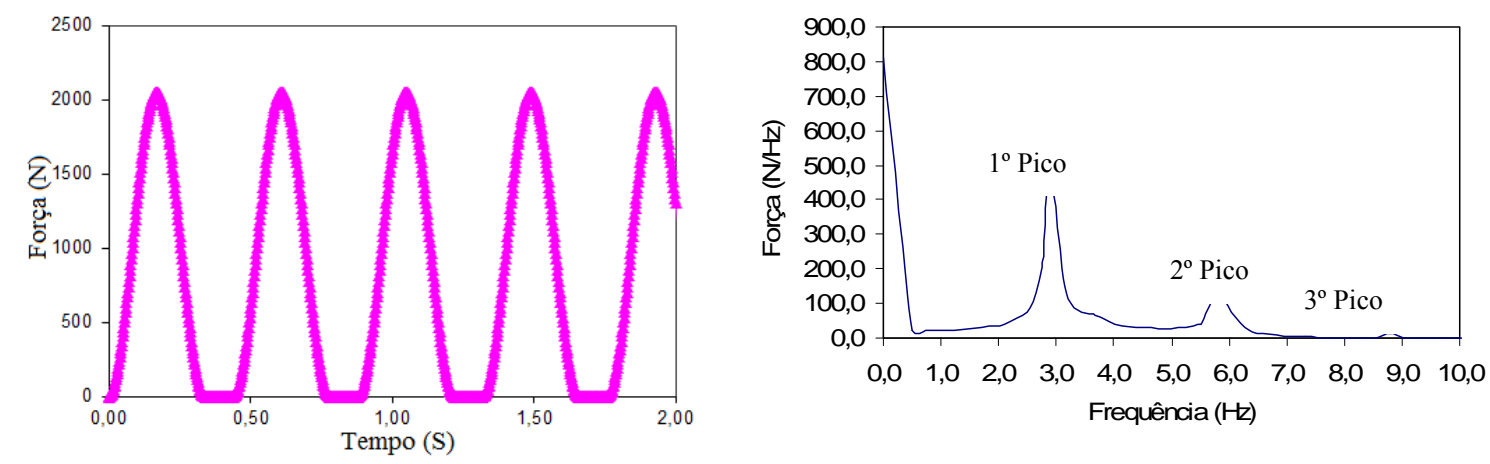

Figura 2. Sinais de força no tempo e espectro de frequência para atividade ginástica aeróbica.

\section{MODELO ESTRUTURAL INVESTIGADO}

O sistema estrutural diz respeito a um piso composto por pilares e vigas de aço e lajes de concreto armado, formando assim um sistema misto. De acordo com as Figuras 3 e 4, o sistema é composto por quatro vãos de $10 \mathrm{~m}$ nas duas direções, perfazendo uma área total de $1600 \mathrm{~m}^{2}$. O espaçamento entre as vigas secundárias é mantido constante e igual a $2,5 \mathrm{~m}$. $\mathrm{O}$ modelo estrutural possui colunas com pé-direito de $4 \mathrm{~m}$. As vigas que se apoiam sobre pilares são ditas vigas principais, e aquelas que se apoiam sobre vigas principais são chamadas de vigas secundárias, Figuras 3 e 4. 


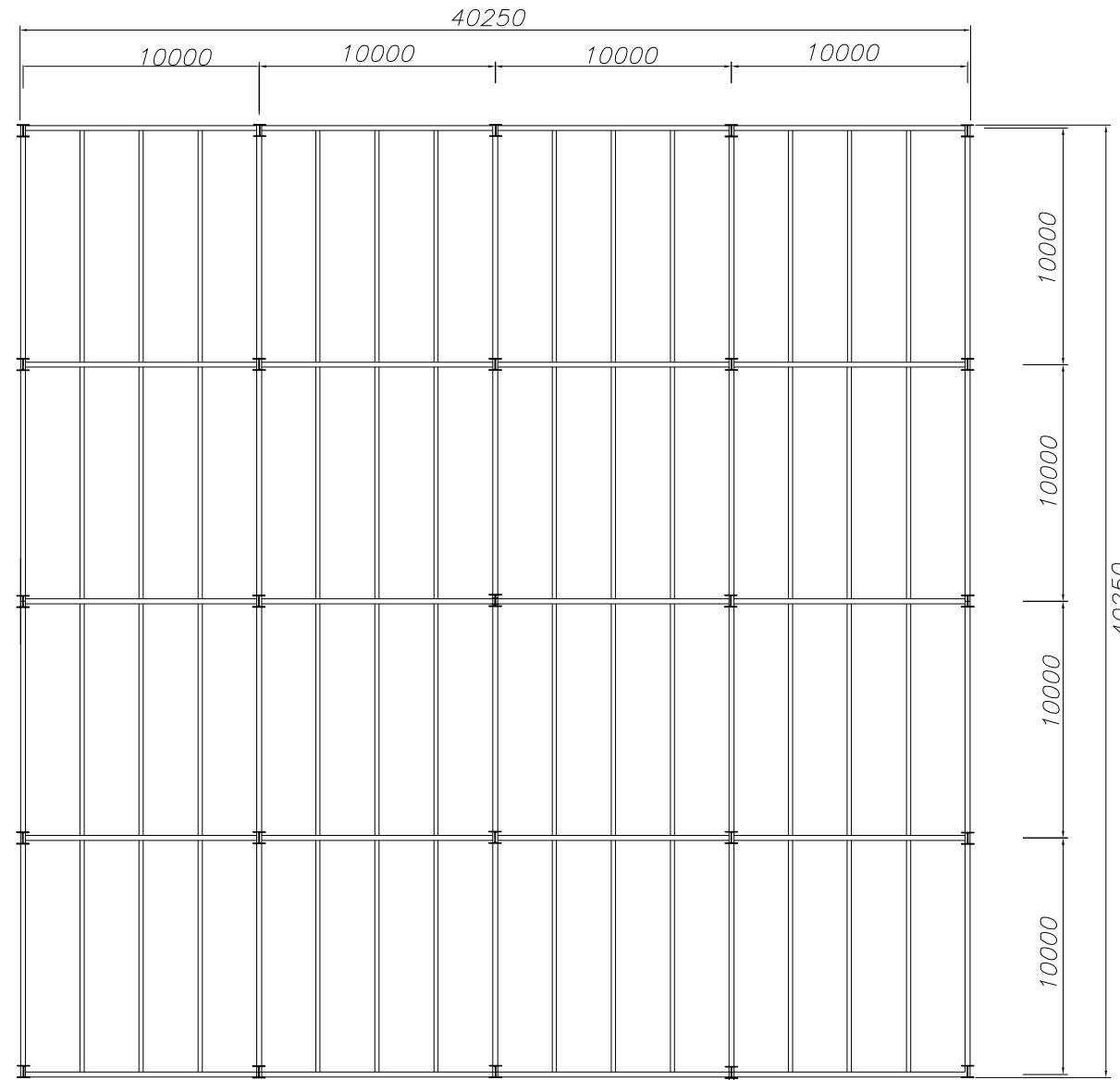

Figura 3. Planta baixa do piso misto (aço-concreto). Cotas em (mm).

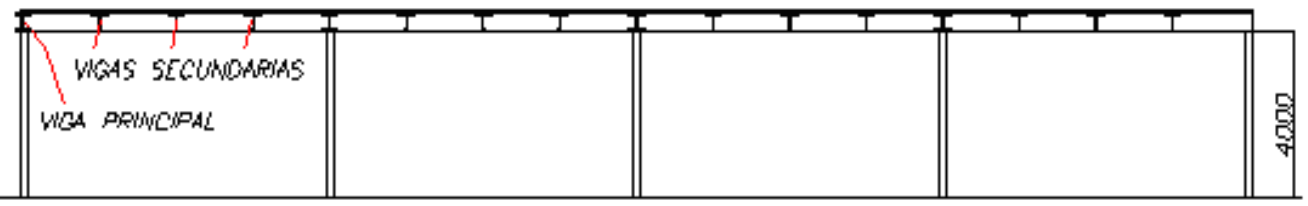

Figura 4. Seção transversal típica. Cotas em (mm).

Os perfis metálicos são do tipo "W", constituídos por um aço com limite de escoamento de $345 \mathrm{MPa}$. Para as vigas de aço foi considerado um módulo de elasticidade igual a 205GPa. A laje de concreto possui espessura de $0,10 \mathrm{~m}$, resistência característica a compressão de 30MPa e módulo de elasticidade igual a 26GPa. A Tabela 3 ilustra as dimensões dos perfis metálicos.

Tabela 3. Características geométricas das vigas e colunas do piso (dimensões em mm)

\begin{tabular}{cccccc}
\hline Tipo de Perfil & $\begin{array}{c}\text { Altura do } \\
\text { Perfil } \\
(\mathrm{d})\end{array}$ & $\begin{array}{c}\text { Largura } \\
\text { da Mesa } \\
\left(\mathrm{b}_{\mathrm{f}}\right)\end{array}$ & $\begin{array}{c}\text { Espessura da } \\
\text { Mesa Superior } \\
\left(\mathrm{t}_{\mathrm{f}}\right)\end{array}$ & $\begin{array}{c}\text { Espessura da } \\
\text { Mesa Inferior } \\
\left(\mathrm{t}_{\mathrm{f}}\right)\end{array}$ & $\begin{array}{c}\text { Espessura } \\
\text { da Alma } \\
\left(\mathrm{t}_{\mathrm{w}}\right)\end{array}$ \\
\hline Viga - W 610 x 140 & 617 & 230 & 22,2 & 22,2 & 13,1 \\
Viga - W 460 x 60 & 455 & 153 & 13,3 & 13,3 & 8,0 \\
Coluna - HP 250 x 85 & 254 & 260 & 14,4 & 14,4 & 14,4 \\
\hline
\end{tabular}


No que tange às ligações estruturais, estas são consideradas como rígidas, semirrígidas e flexíveis. As ligações semirrígidas e flexíveis são consideradas apenas entre as vigas principais e secundárias (ligações viga-viga). As ligações entre as vigas principais e as colunas são do tipo rígidas. A Figura 5 ilustra a curva momento fletor versus rotação das ligações semirrígidas viga-viga [10]. A rigidez inicial empregada para a modelagem da Semi-rigidez destas ligações foi da ordem de $12 \mathrm{kNmm} / \mathrm{rad}(12000 \mathrm{Nm} / \mathrm{mrad})$.

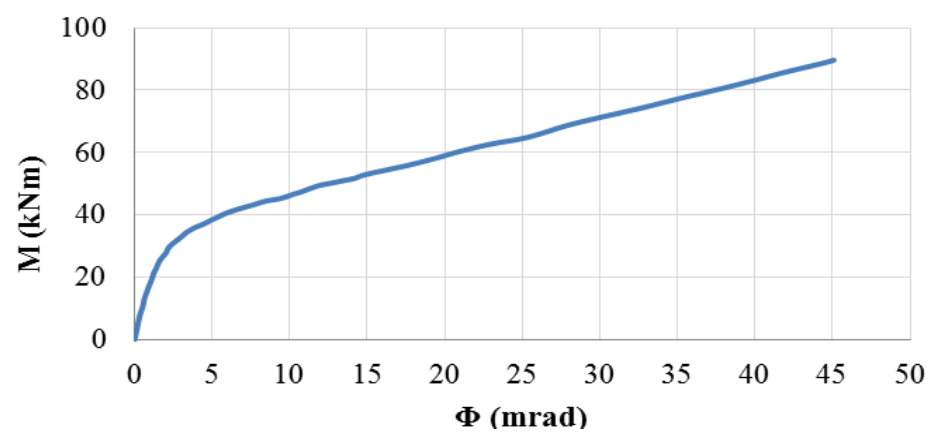

Figura 5. Curva momento versus rotação das ligações semirrígidas [10].

Tendo como o objetivo o estudo sobre a influência do nível de interação aço-concreto (total e parcial) sobre a resposta dinâmica não linear de pisos mistos, foram considerados, neste estudo, dois tipos de conectores de cisalhamento do tipo studs. A Figura 6 apresenta as curvas força versus deslizamento, obtidas a partir de ensaios experimentais [7,11], no que diz respeito aos dois tipos de conectores de cisalhamento empregados neste trabalho de pesquisa.

A Tabela 4 apresenta o número de conectores utilizado em cada viga de aço, de forma a se garantir a compatibilidade de deformações entre os nós dos elementos de casca, representativos da laje de concreto e os elementos de viga tridimensionais, simulando o comportamento de um sistema estrutural misto (aço-concreto), com interação total.

Tabela 4. Características quantidade de conectores utilizados

\begin{tabular}{|c|c|c|c|c|c|}
\hline \multirow[b]{2}{*}{$\begin{array}{l}\text { Tipo do } \\
\text { Conector }\end{array}$} & \multirow[b]{2}{*}{$\begin{array}{c}\mathrm{f}_{\mathrm{ck}} \\
(\mathrm{MPa})\end{array}$} & \multirow[b]{2}{*}{$\begin{array}{l}\text { Rigidez Inicial } \\
\qquad(\mathrm{kN} / \mathrm{mm})\end{array}$} & \multirow[b]{2}{*}{$\begin{array}{l}\text { Referências } \\
\text { Utilizadas }\end{array}$} & \multicolumn{2}{|c|}{ Número de Conectores por Viga } \\
\hline & & & & $\begin{array}{c}\text { Vigas } \\
\text { Secundárias }\end{array}$ & $\begin{array}{c}\text { Vigas } \\
\text { Principais }\end{array}$ \\
\hline Stud $13 \mathrm{~mm}$ & 20 & 65 & [7] & 448 & 720 \\
\hline Stud 19mm & 34,6 & 200 & [11] & 200 & 320 \\
\hline
\end{tabular}

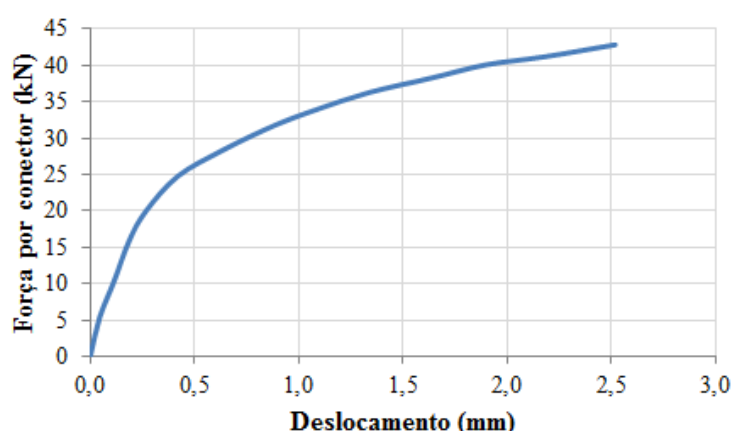

a) Conector do tipo Stud $13 \mathrm{~mm}[7]$.

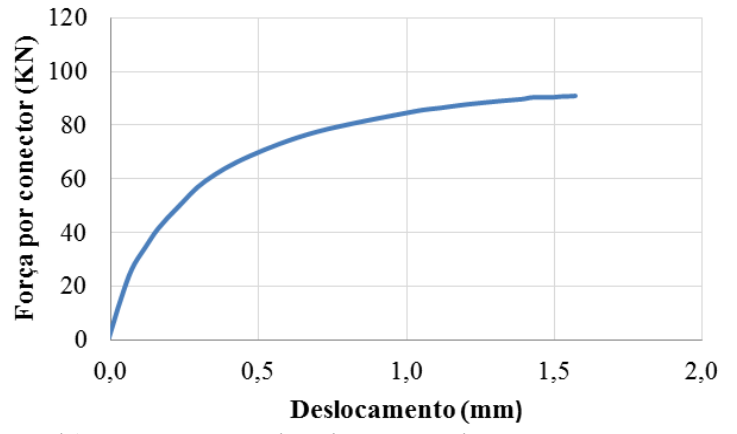

b) Conector do tipo Stud 19mm [11].

Figura 6. Curva força x deslizamento dos conectores de cisalhamento. 
A Figura 7 ilustra o esquema em planta, associado à distribuição das pessoas praticando ginástica aeróbica sobre o piso em estudo (Pontos A-D, Figura 7) e, ainda, seu posicionamento sobre a estrutura, objetivando facilitar o entendimento e a visualização dos pontos de carregamento e dos pontos onde a resposta dinâmica foi obtida. Ressalta-se que a resposta dinâmica da estrutura é obtida em todos os pontos (Pontos A-H, Figura 7).

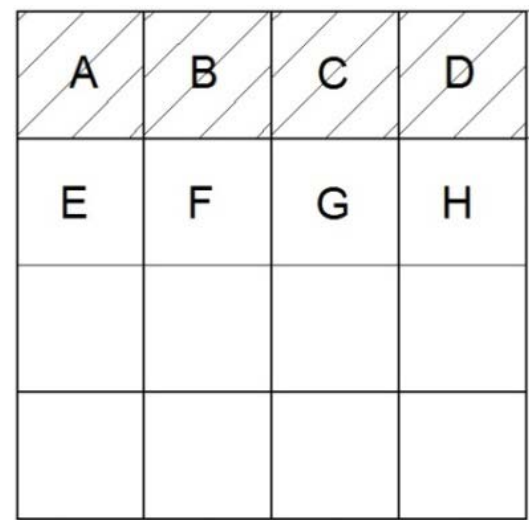

a) Distribuição e posicionamento das cargas

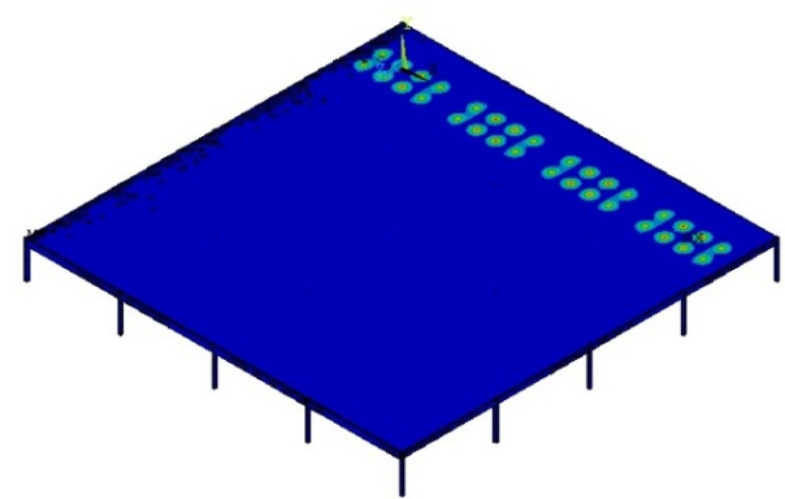

b) Posicionamento do carregamento [8]

Figura 7. Distribuição das cargas dinâmicas sobre o piso (32 pessoas).

\section{MODELO NUMÉRICO-COMPUTACIONAL}

Os modelos numérico-computacionais foram desenvolvidos utilizando-se técnicas usuais de discretização, via método dos elementos finitos, por meio do emprego do programa Ansys [8]. As vigas e as colunas de aço dos modelos estruturais são simuladas por elementos finitos de viga tridimensionais BEAM44 [8], onde os efeitos de flexão e torção são considerados. A laje de concreto é simulada por meio de elementos finitos de casca SHELL63 [8].

Para a modelagem das ligações viga-viga flexíveis (ligações rotuladas), utilizou-se o elemento COMBIN7 [8], e para a simulação das ligações viga-viga semirrígidas foi empregado o elemento COMBIN39 [8]. Finalmente, a estratégia de modelagem utiliza os elementos de mola rotacional, COMBIN39 [8], para representar o comportamento dos conectores de cisalhamento do sistema estrutural real.

Considera-se, ainda, que os materiais empregados nas estruturas em estudo, aço e concreto, trabalhem no regime linear-elástico. Na sequência do texto, A Figura 8 ilustra os elementos finitos empregados para a modelagem do sistema estrutural e a Figura 9 ilustra as malhas de elementos finitos utilizados ao longo da presente investigação.

\section{ANÁLISE DINÂMICA DA ESTRUTURA}

A resposta dinâmica do piso é determinada, inicialmente, mediante a obtenção das frequências naturais e modos de vibração. Inicialmente, considera-se o piso trabalhando em regime de interação parcial e total e, em seguida, a variação do número de conectores é considerada. Com o objetivo de avaliar o nível de conforto humano do modelo, de acordo com a metodologia de análise proposta, são obtidos, também, os valores das acelerações de pico. 

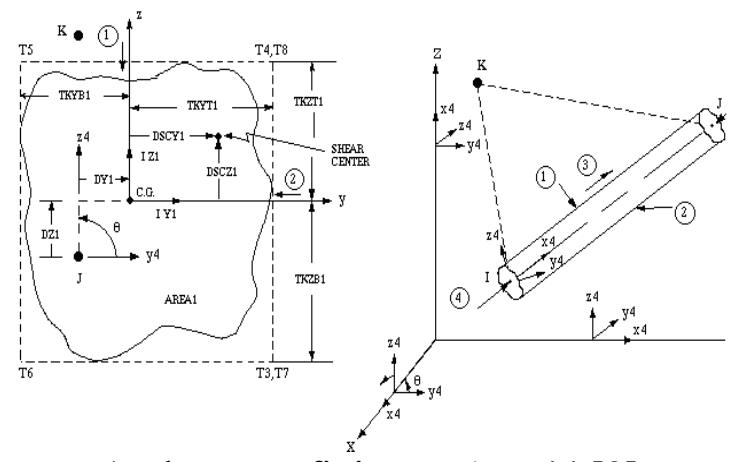

a) Elemento finito BEAM 44 [8].

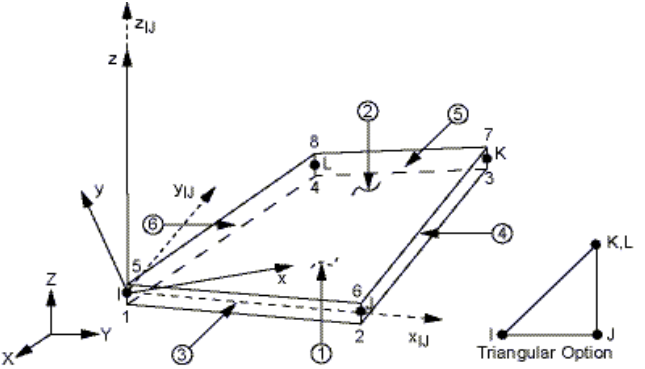

b) Elemento finito SHELL63 [8].

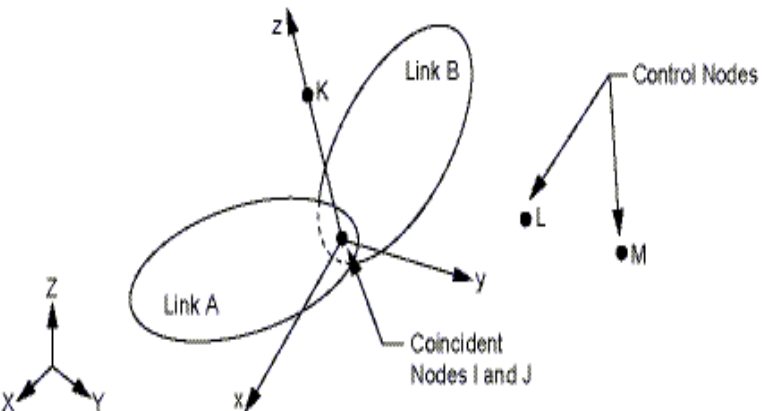

c) Elemento finito COMBIN7 [8].

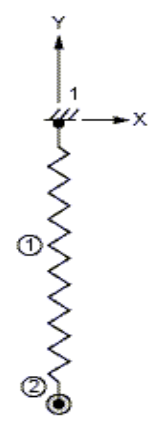

d) Elemento finito COMBIN39 [8].

Figura 8. Elementos finitos utilizados na discretização do modelo.

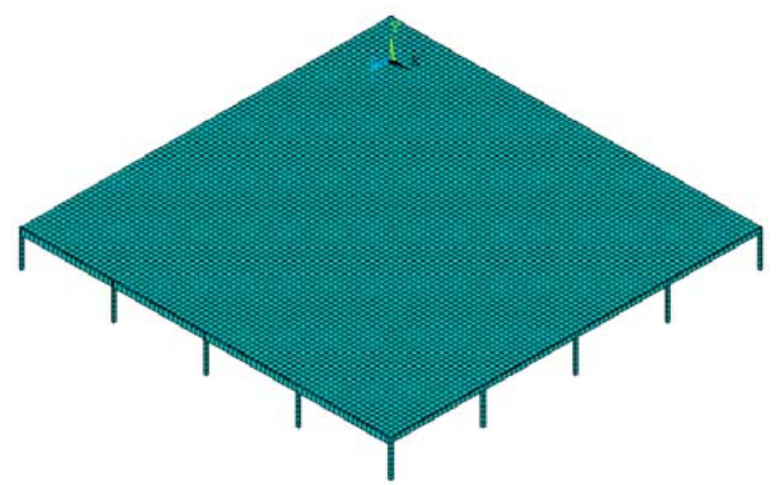

a) Vista Tridimensional.

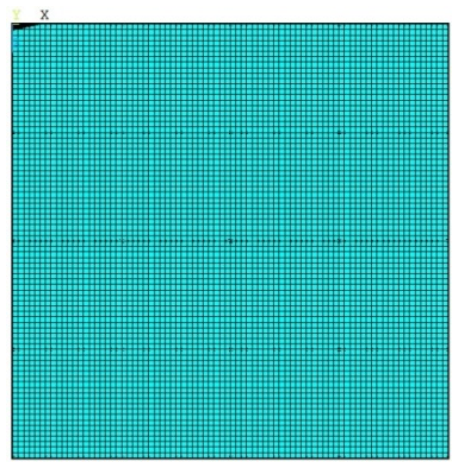

b) Vista Superior.

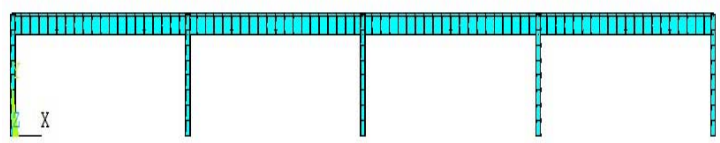

c) Plano XY.

\begin{tabular}{|ll|}
\hline Elementos & $=32036$ \\
Beam44 & $=3920$ \\
Shell63 & $=25600$ \\
Combin39 & $=2516$ \\
Nós & $=29874$ \\
Graus de Liberdade & $=166589$
\end{tabular}

Figura 9. Malhas de elementos finitos representativas do modelo estrutural. 


\subsection{Análise das frequências naturais e dos modos de vibração dos modelos}

Através das análises de vibração livre do modelo estrutural realizadas pelo programa computacional Ansys [8], foram obtidos os valores das frequências naturais (autovalores) e os respectivos modos de vibração (autovetores). As Tabelas 5 e 6 apresentam os valores das seis primeiras frequências naturais do modelo estrutural misto estudado. Desta forma, torna-se possível observar a influência das ligações estruturais e, bem como, a influência das interações parcial e total, sobre o comportamento dinâmico do modelo estrutural em estudo.

Tabela 5. Frequências naturais do modelo com Stud $13 \mathrm{~mm}\left(\mathrm{~S}_{\mathrm{j}}=65 \mathrm{kN} / \mathrm{mm}\right)$

\begin{tabular}{ccccccc}
\hline Frequência & \multicolumn{3}{c}{ Interação Total } & \multicolumn{3}{c}{ Interação Parcial (50\%) } \\
\cline { 2 - 7 }$(\mathrm{Hz})$ & Rígido & Semirrígido & Flexível & Rígido & Semirrígido & Flexível \\
\hline $\mathrm{f}_{01}$ & 6,49 & 6,08 & 5,93 & 6,32 & 5,91 & 5,76 \\
$\mathrm{f}_{02}$ & 6,61 & 6,33 & 6,22 & 6,45 & 6,19 & 6,05 \\
$\mathrm{f}_{03}$ & 6,95 & 6,44 & 6,50 & 6,76 & 6,27 & 6,31 \\
$\mathrm{f}_{04}$ & 6,95 & 6,63 & 6,50 & 6,77 & 6,46 & 6,31 \\
$\mathrm{f}_{05}$ & 7,02 & 6,87 & 6,76 & 6,87 & 6,72 & 6,58 \\
$\mathrm{f}_{06}$ & 7,16 & 6,99 & 6,88 & 7,01 & 6,83 & 6,68 \\
$\mathrm{f}_{07}$ & 7,21 & 7,05 & 6,93 & 7,03 & 6,89 & 6,73 \\
\hline
\end{tabular}

Tabela 6. Frequências naturais do modelo com Stud $19\left(\mathrm{~S}_{\mathrm{j}}=200 \mathrm{kN} / \mathrm{mm}\right)$

\begin{tabular}{ccccccc}
\hline Frequência & \multicolumn{3}{c}{ Interação Total } & \multicolumn{3}{c}{ Interação Parcial $(50 \%)$} \\
\cline { 2 - 7 }$(\mathrm{Hz})$ & Rígido & Semirrígido & Flexível & Rígido & Semirrígido & Flexível \\
\hline $\mathrm{f}_{01}$ & 6,55 & 6,11 & 5,98 & 6,39 & 5,98 & 5,84 \\
$\mathrm{f}_{02}$ & 6,67 & 6,38 & 6,27 & 6,52 & 6,26 & 6,13 \\
$\mathrm{f}_{03}$ & 7,01 & 6,50 & 6,35 & 6,84 & 6,35 & 6,19 \\
$\mathrm{f}_{04}$ & 7,02 & 6,69 & 6,56 & 6,85 & 6,54 & 6,40 \\
$\mathrm{f}_{05}$ & 7,22 & 6,92 & 6,81 & 6,94 & 6,79 & 6,67 \\
$\mathrm{f}_{06}$ & 7,22 & 7,05 & 6,94 & 7,08 & 6,91 & 6,78 \\
$\mathrm{f}_{07}$ & 7,27 & 7,10 & 6,99 & 7,11 & 6,97 & 6,83 \\
\hline
\end{tabular}

Observando-se os valores apresentados nas Tabelas 5 e 6, percebe-se, claramente, uma redução nos valores das frequências naturais dos modelos estruturais quando os modelos flexíveis e semirrígidos são comparados com os modelos estruturais rígidos. Observa-se, ainda, uma pequena redução nos valores das frequências naturais quando o modelo com interação total é comparado em relação ao modelo com interação parcial. Em termos absolutos, foi observado que existe uma pequena diminuição nos valores das frequências, a partir da diminuição do número e, também, da rigidez inicial dos conectores, Tabelas 5 e 6.

De forma geral, a redução nas frequências naturais torna o piso ainda mais suscetível às excitações dinâmicas induzidas pelos seres humanos e, portanto, a influência das interações (total e parcial) e das ligações estruturais sobre a resposta dinâmica dos pisos mistos (açoconcreto) deve merecer atenção especial por parte dos projetistas de estruturas metálicas. $\mathrm{Na}$ sequência a Figura 10 apresenta as formas modais referentes ao modelo estrutural em estudo, segundo níveis de interação total e parcial. 


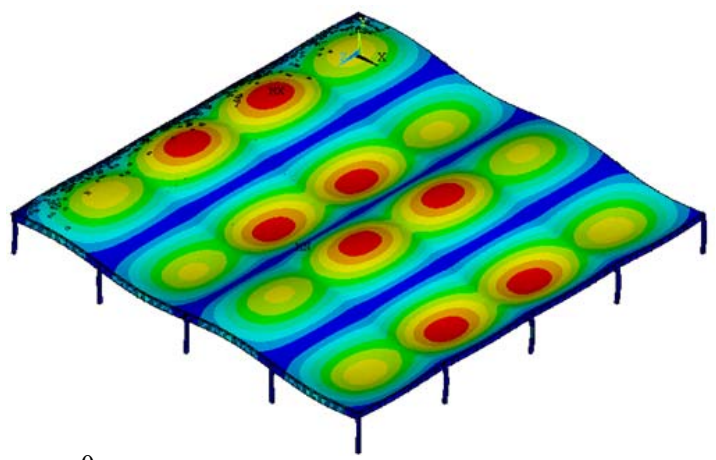

a) $1^{0}$ Modo de vibração $\left(\mathrm{f}_{01}=6,08 \mathrm{~Hz}\right)$. Ligações semirrígidas. Interação total.

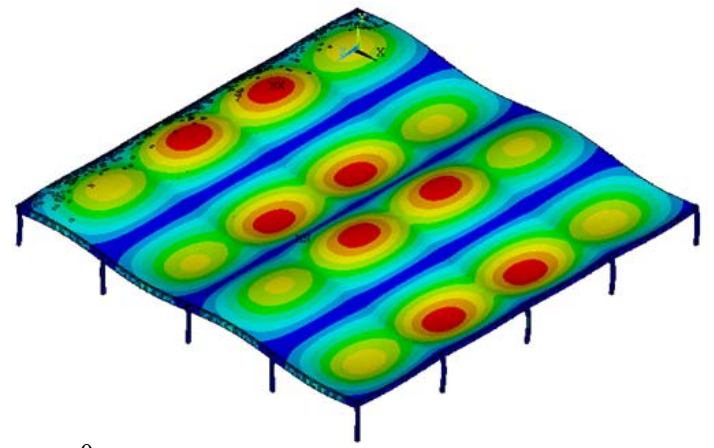

c) $1^{0}$ Modo de vibração $\left(\mathrm{f}_{01}=5,91 \mathrm{~Hz}\right)$. Ligações semirrígidas. Interação parcial.

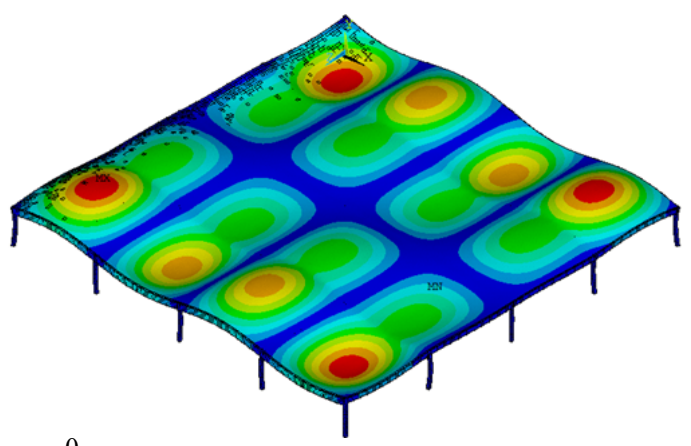

b) $2^{0}$ Modo de vibração $\left(\mathrm{f}_{02}=6,33 \mathrm{~Hz}\right)$. Ligações semirrígidas. Interação total.

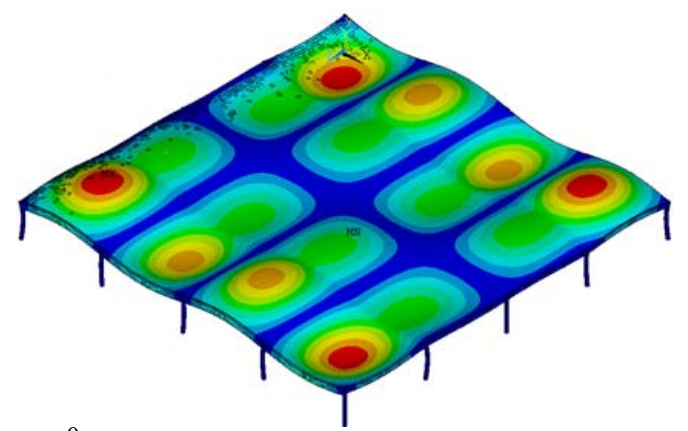

d) $2^{0}$ Modo de vibração $\left(\mathrm{f}_{02}=5,19 \mathrm{~Hz}\right)$. Ligações semirrígidas. Interação parcial.

Figura 10. Modos de vibração do modelo estrutural (interação total e parcial).

Observando a Figura 10, verifica-se, com clareza, que os modos de vibração do sistema estrutural analisado, independentemente do grau interação considerada (total ou parcial) e das ligações estruturais (rígidas, semirrígidas ou flexíveis), apresentam predominância dos efeitos de flexão, no que tange ao primeiro modo de vibração (modo fundamental de vibração) e de flexo-torção, no que diz respeito ao segundo modo de vibração do piso.

\subsubsection{Variações das frequências de acordo com o número de conectores}

Estudou-se, nesta fase, a variação do número de conectores de cisalhamento no modelo e a sua influência na rigidez da estrutura. A estratégia de análise aqui realizada foi utilizar as curvas força deslizamento obtida experimentalmente por [7, 11]. Assim sendo, os gráfícos da Figura 11 apresentam os valores das frequências naturais obtidas a partir da variação do número de conectores de cisalhamento de acordo com [4].

Observando-se os gráficos apresentados na Figura 11, verifica-se que em ambos os modelos (interação parcial: Stud de $13 \mathrm{~mm}$ e Stud de $19 \mathrm{~mm}$ ), existe uma variação aproximadamente linear (frequência versus grau de interação) na medida em que se modifica o número de conectores de cisalhamento. Ou seja: os valores das frequências naturais da estrutura tendem a diminuir na medida em que se diminui o número de conectores, o que torna os pisos mais flexíveis e, portanto, mais suscetíveis a vibrações ocasionadas por pequenos impactos. Isso decorre do fato de que o número de conectores do sistema misto influencia diretamente a rigidez global do piso. 


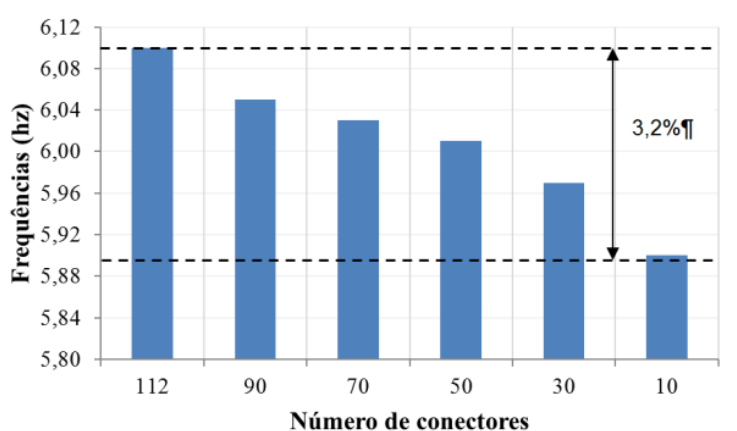

a) Variação dos conectores das vigas secundárias. Stud $13 \mathrm{~mm} . \mathrm{S}_{\mathrm{j}}=65 \mathrm{kN} / \mathrm{mm}$.

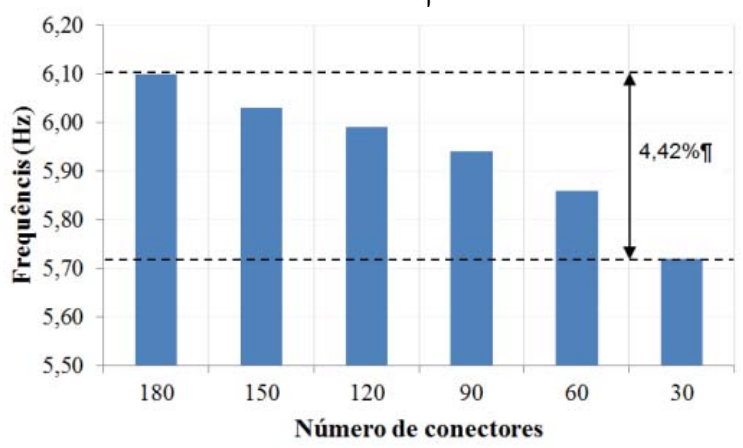

c) Variação dos conectores das vigas principais. Stud $13 \mathrm{~mm}$. $\mathrm{S}_{\mathrm{j}}=65 \mathrm{kN} / \mathrm{mm}$.

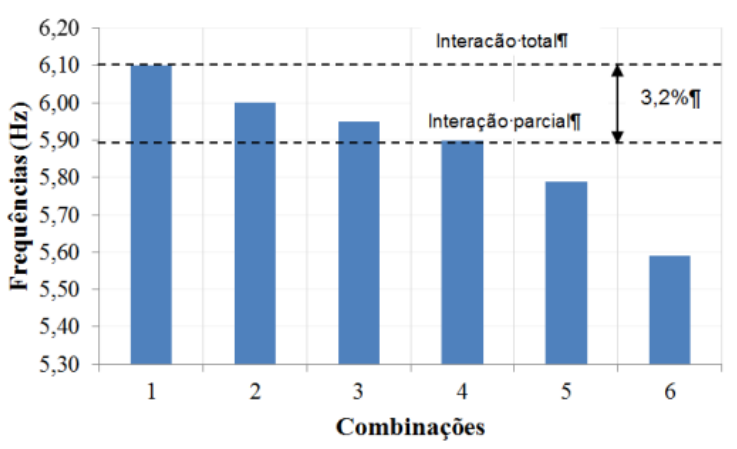

e) Combinação das vigas principais e secundárias. Stud $13 \mathrm{~mm} . \mathrm{S}_{\mathrm{j}}=65 \mathrm{kN} / \mathrm{mm}$.

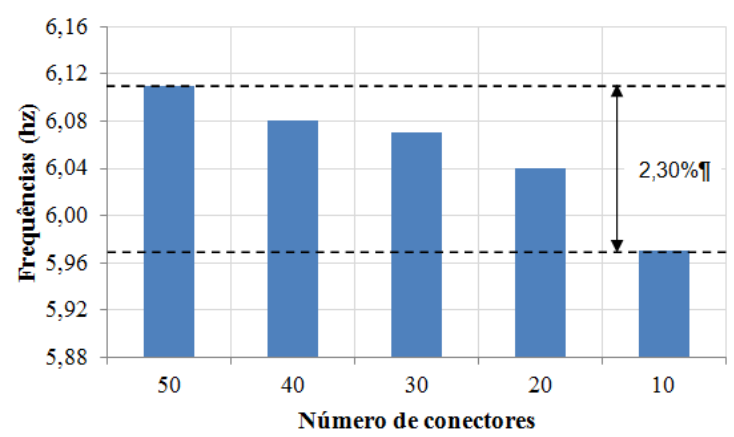

b) Variação dos conectores das vigas secundárias. Stud $19 \mathrm{~mm}$. $\mathrm{S}_{\mathrm{j}}=200 \mathrm{kN} / \mathrm{mm}$.

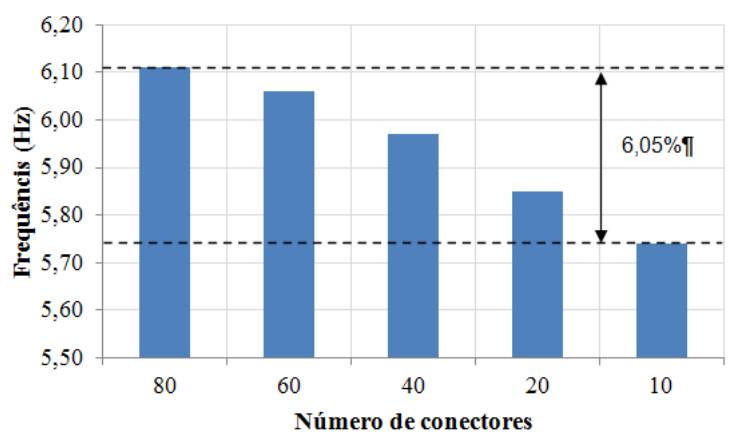

d) Variação dos conectores das vigas principais. Stud $19 \mathrm{~mm} . \mathrm{S}_{\mathrm{j}}=200 \mathrm{kN} / \mathrm{mm}$.

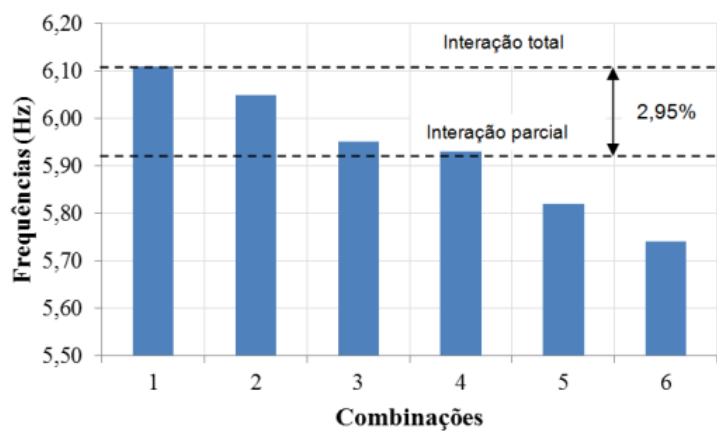

f) Combinação das vigas principais e secundárias. Stud $19 \mathrm{~mm}$. $\mathrm{S}_{\mathrm{j}}=200 \mathrm{kN} / \mathrm{mm}$.

Figura 11. Frequências naturais em função da variação do número de conectores

Por outro lado, em termos quantitativos, pode-se verificar que a diferença percentual máxima nos valores das frequências naturais quando são comparados os níveis de interação aço-concreto (interação total versus interação parcial) é da ordem de $5 \%$, portanto uma diferença muito pequena.

\subsection{Estudo sobre o conforto humano do piso misto (aço-concreto)}

Na sequência do estudo, as Figuras 12 e 13 apresentam a resposta dinâmica do piso misto, ao longo do tempo, em termo dos deslocamentos translacionais verticais e das acelerações máximas. A resposta do modelo estrutural foi obtida no centro do painel das lajes de concreto (Figura 7). Estas grandezas (deslocamentos e acelerações) foram obtidas considerando-se trinta e duas pessoas praticando ginástica aeróbica sobre o piso, em posições simétricas 
em relação centro do referido painel (Figura 7), e acordo com uma taxa de distribuição de 0,25 pessoas $/ \mathrm{m}^{2}[9]$.

Destaca-se que no lado esquerdo das Figura 12 e 13 são apresentados os gráficos dos deslocamentos translacionais verticais e na parte a direita às respectivas acelerações. Convém chamar a atenção do leitor, para o fato de que os valores das acelerações de pico foram obtidos na fase permanente da resposta do sistema. Figuras 12 e 13, quando submetidos à ação de trinta e duas pessoas praticando ginástica aeróbica sobre o piso.
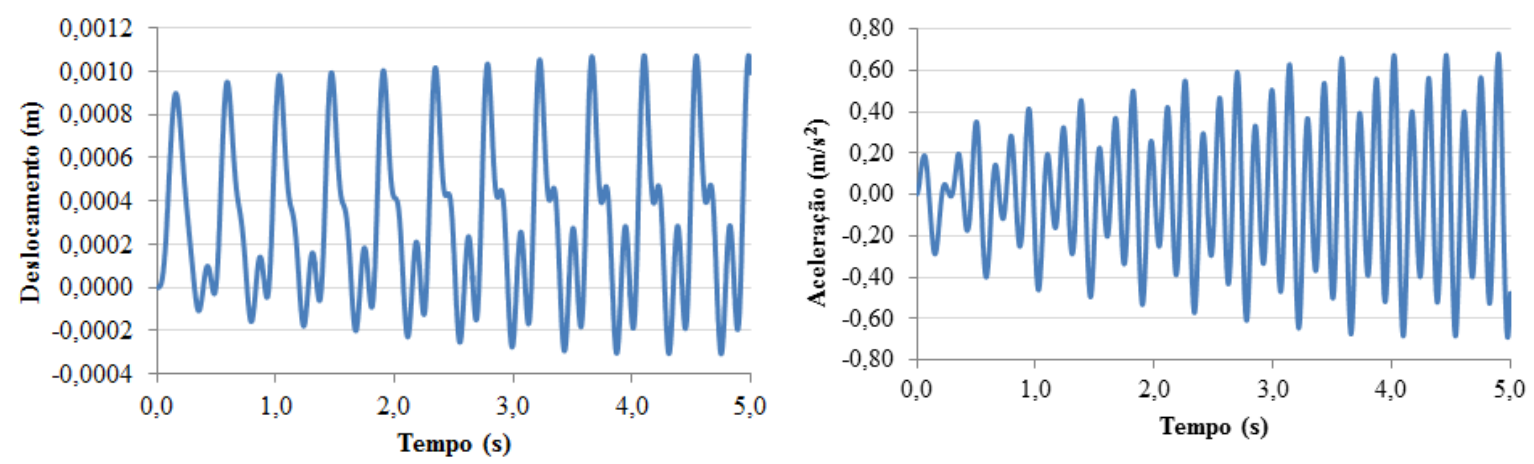

Figura 12. Deslocamentos e acelerações. Ligações semirrígidas. Interação Parcial: Nó A
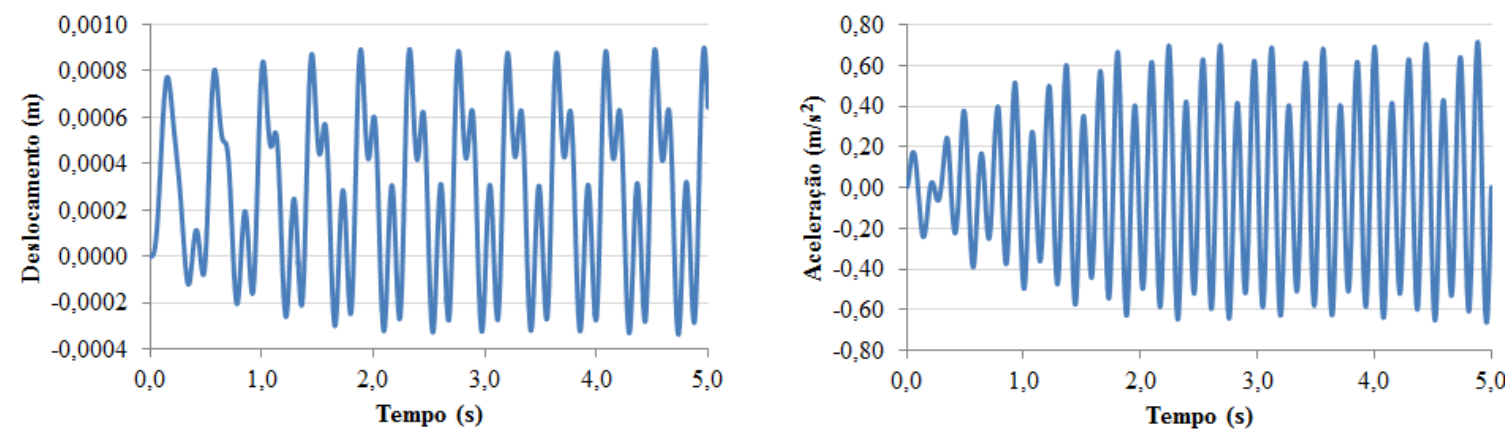

Figura 13. Deslocamentos e acelerações. Ligações semirrígidas. Interação Parcial: Nó B.

As Figuras 12 e 13 ilustram a variação dos deslocamentos translacionais verticais e acelerações, ao longo do tempo, no que tange ao nó A e B do modelo estrutural, considerando-se o grau de interação parcial (50\%) entre o aço e o concreto, para trinta e duas pessoas, praticando ginástica aeróbica. O maior pico de aceleração foi da ordem de $0,8 \mathrm{~m} / \mathrm{s}^{2}$, sobre o piso misto (aço-concreto), com ligações viga-viga flexíveis e frequência fundamental igual a $5,90 \mathrm{~Hz}$.

Com base nos valores de aceleração de pico encontrados, correspondente a fase permanente da resposta do modelo estrutural estudado, foi possível observar que estes valores são mais elevados quando se considera o efeito da interação parcial e quando as ligações vigaviga do piso misto (aço-concreto) são consideradas como flexíveis ou semirrígidas. Onde na maioria dos casos, com trinta e duas pessoas praticando ginástica aeróbica, as acelerações de pico ultrapassam os limites para conforto humano, de acordo com normas e recomendações de projeto $\left(\mathrm{a}_{\lim }=0,50 \mathrm{~m} / \mathrm{s}^{2}\right)[6,12]$.

Na sequência do texto, as Tabelas 7 e 8 apresentam os valores das acelerações de pico 
para o caso de carregamento dinâmico com trinta e duas pessoas praticando ginástica aeróbica considerando o efeito da interação total e interação parcial.

Tabela 7: Acelerações de pico. Carregamento: 32 pessoas: Nós A, B, C e D (ver Figura 7)

\begin{tabular}{cccccc}
\hline \multirow{2}{*}{ Interação } & \multirow{2}{*}{ Modelo } & $\begin{array}{c}\mathrm{a}_{\mathrm{p}}\left(\mathrm{m} / \mathrm{s}^{2}\right) \\
\text { Nó A }\end{array}$ & $\begin{array}{c}\mathrm{a}_{\mathrm{p}}\left(\mathrm{m} / \mathrm{s}^{2}\right) \\
\text { Notal }\end{array}$ & $\begin{array}{c}\mathrm{a}_{\mathrm{p}}\left(\mathrm{m} / \mathrm{s}^{2}\right) \\
\text { Nó C }\end{array}$ & $\begin{array}{c}\mathrm{a}_{\mathrm{p}}\left(\mathrm{m} / \mathrm{s}^{2}\right) \\
\text { Nó D }\end{array}$ \\
\hline \multirow{4}{*}{ Parcial (50\%) } & Rígido & 0,26 & 0,17 & 0,17 & 0,26 \\
& Semirrígido & 0,28 & 0,20 & 0,20 & 0,28 \\
& Flexível & 0,30 & 0,44 & 0,43 & 0,30 \\
& Rígido & 0,60 & 0,33 & 0,33 & 0,60 \\
& Semirrígido & 0,61 & 0,62 & 0,62 & 0,61 \\
Limite de aceleração: $\mathrm{a}_{\lim }=0,5 \mathrm{~m} / \mathrm{s}^{2}[6,12]$ & 0,64 & 0,80 & 0,80 & 0,64 \\
\hline
\end{tabular}

Tabela 8: Acelerações de pico. Carregamento: 32 pessoas: Nós E, F, G e H (ver Figura 7)

\begin{tabular}{cccccc}
\hline \multirow{2}{*}{ Interação } & \multirow{2}{*}{ Modelo } & $\mathrm{a}_{\mathrm{p}}\left(\mathrm{m} / \mathrm{s}^{2}\right)$ & $\mathrm{a}_{\mathrm{p}}\left(\mathrm{m} / \mathrm{s}^{2}\right)$ & $\mathrm{a}_{\mathrm{p}}\left(\mathrm{m} / \mathrm{s}^{2}\right)$ & $\mathrm{a}_{\mathrm{p}}\left(\mathrm{m} / \mathrm{s}^{2}\right)$ \\
& & Nó E & Nó F & Nó G & Nó H \\
\hline \multirow{3}{*}{ Total } & Rígido & 0,035 & 0,035 & 0,035 & 0,035 \\
& Semirrígido & 0,087 & 0,036 & 0,036 & 0,087 \\
& Flexível & 0,088 & 0,09 & 0,09 & 0,088 \\
& Rígido & 0,28 & 0,13 & 0,13 & 0,28 \\
& Semirrígido & 0,30 & 0,14 & 0,14 & 0,30 \\
& Flexível & 0,32 & 0,23 & 0,23 & 0,32 \\
\hline
\end{tabular}

Limite de aceleração: $\mathrm{a}_{\lim }=0,5 \mathrm{~m} / \mathrm{s}^{2}[6,12]$

Foi observado ao longo do estudo um crescimento nos valores das acelerações de pico do modelo quando são comparados os modelos compostos por ligações viga-viga semirrígidas e flexíveis em relação aqueles em que as ligações viga-viga são rígidas.

Percebe-se, claramente, um crescimento nos valores das acelerações de pico (Tabelas 7 e 8), quando são comparados os modelos em que se considera o efeito da interação parcial $(50 \%)$ em relação aqueles com interação total $\left(a_{p}=0,80 \mathrm{~m} / \mathrm{s}^{2}\right.$ : interação parcial com ligações flexíveis entre as vigas e $a_{p}=0,44 \mathrm{~m} / \mathrm{s}^{2}$ : interação total com ligações flexíveis entre as vigas). Verifica-se, também, que, quando se considera o efeito da interação parcial, praticamente em todos os casos estudados, os critérios estabelecidos para conforto humano são violados, de acordo com normas e recomendações de projeto. $\left(\mathrm{a}_{\lim }=0,50 \mathrm{~m} / \mathrm{s}^{2}\right)[6,12]$.

\section{CONCLUSÕES}

Esta investigação apresenta os resultados de um estudo desenvolvido para avaliar-se o comportamento estrutural de pisos mistos (aço-concreto), quando submetidos a excitações dinâmicas humanas rítmicas. Para tal foi desenvolvido um modelo computacional, com base nos elementos finitos, por meio do programa Ansys [8], que permite a modelagem do sistema estrutural de acordo com o nível de interação aço-concreto (interação total ou parcial) e incorpora o efeito das ligações viga-viga e viga-coluna (ligações rígidas, semirrígidas e flexíveis).

A metodologia de análise proposta considera o estudo do comportamento dinâmico 
não linear em regime de serviço de pisos mistos (aço-concreto). Assim sendo, foi possível avaliar o nível de vibração dos pisos, quando submetidos à ginástica aeróbica, com base na obtenção da resposta dinâmica não linear dos modelos, em termos das acelerações de pico.

Em relação à influência dos conectores utilizados nesta investigação, verifica-se que, em termos absolutos, ocorre uma diminuição nos valores das frequências naturais do piso, de acordo com a diminuição do número de conectores e do valor da rigidez inicial destes. Todavia, em termos quantitativos, pode-se verificar que a diferença percentual máxima nos valores das frequências naturais quando são comparados os níveis de interação aço-concreto (interação total versus interação parcial) é da ordem de 5\%, portanto uma diferença muito pequena.

Observando-se os valores das acelerações de pico obtidas ao longo da análise, percebe-se que o tipo de conector utilizado para simulação da interação parcial de $50 \%$ (studs), para o piso investigado e para os valores de rigidez inicial adotadas, não alterou estes valores de forma significativa. A pesquisa precisa avançar, principalmente, no que diz respeito à variação da rigidez inicial dos conectores e, bem como, no que diz respeito à modelagem da interação parcial (aço-concreto). Por outro lado, foi verificado que as ligações entre as vigas (ligações viga-viga), para o piso investigado, alteram de forma mais significativa os valores finais das acelerações de pico. O que é um indicativo de que os critérios de projeto devem levar em conta o caráter dinâmico da excitação no que tange a verificação os estados limites de utilização (vibrações excessivas).

Para os casos de carregamento dinâmico considerados nesta investigação foi observado que o modelo estrutural em questão viola os critérios estabelecidos para conforto humano, de acordo com normas e recomendações de projeto. Deste modo sugere-se a continuação desta pesquisa com base no desenvolvimento de extensas análises paramétricas, através da modificação de parâmetros de projeto, tais como: modelagem das ligações estruturais e da interação parcial, espessura da laje de concreto, características geométricas das vigas e colunas de aço. Aspectos referentes à variação dos parâmetros adotados na função representativa do carregamento dinâmico também necessitam de investigação no estudo paramétrico.

\section{Agradecimentos}

Os autores agradecem ao suporte financeiro fornecido pelas Agências de Fomento à Pesquisa do país: CAPES, CNPq e FAPERJ, que possibilitaram a realização deste trabalho de pesquisa.

\section{REFERÊNCIAS}

[1] Castro R. A., "Modelagem Computacional de Ligações Semirrígidas e sua Influência na Resposta Dinâmica Não-Linear de Pórticos de Aço", Dissertação de Mestrado - PGECIV/ UERJ, Rio de Janeiro, Brasil, 2006.

[2] Faísca R. G., "Caracterização de Cargas Dinâmicas Geradas por Atividades Humanas", 230 páginas, Tese de Doutorado - COPPE/UFRJ, Rio de Janeiro, RJ, Brasil, 2003.

[3] Gonçalves S. G., “Análise dinâmica não linear de pisos mistos submetidos a atividades 
humanas rítmicas”, Dissertação de Mestrado, - PGECIV/UERJ, Rio de Janeiro/RJ, Brasil, 2011.

[4] Lopes, E. D. C., "Efeito das ligações viga-coluna e viga-viga e dos conectores de cisalhamento na interação aço-concreto dos pisos mistos". Tese de Doutorado, DEC/PUC-RIO, Rio de Janeiro/RJ, Brasil, 2012.

[5] Mello A. V. de A., "Vibrações em pisos de edificações induzidas por atividades humanas”, Dissertação de Mestrado, PGECIV/UERJ, Rio de Janeiro/RJ, Brasil, 2005.

[6] Murray T. M., Allen D. E., Ungar E. E., "Floor Vibrations Due to Human Activity", Steel Design Guide Series, American Institute of Steel Construction, AISC, 2003.

[7] Tristão G. A., "Comportamento de conectores de cisalhamento em vigas mistas açoconcreto com análise da resposta numérica", Dissertação Mestrado. Escola de Engenharia de São Carlos, Universidade de São Paulo, São Carlos, 2002.

[8] Ansys, "Swanson Analysis Systems, Inc., P,O, Box 65, Johnson Road, Houston, PA, 15342-0065”, Version 10,0, Basic analysis procedures, 2nd Edition, 2003.

[9] Bachmann H., Ammann W., "Vibrations in structures induced by man and machines", IABSE Structural Engineering Document 3E, International Association for Bridges and Structural Engineering, ISBN 3-85748-052-X, 1987.

[10] Oliveira T. J. L., "Pisos Mistos Aço-Concreto com Ligações Semirrígidas sob Ação de Cargas de Gravidade: Analise Estática Não-Linear e Dinâmica, Experimental e numérica", Tese de Doutorado, COPPE/UFRJ, Rio de Janeiro, RJ, Brasil, 2007.

[11] Ellobody E., Young B., "Performance of shear connection in composite beans with profiled steel sheeting", Journal of constructional steel research, V,62, P,682-694, NOV, 2005.

[12] International Standards Organisation / ISO 2631-2. "Evaluation of Human Exposure to Whole-Body Vibration", Continuous and Shock-Induced Vibration in Buildings (1-80Hz), 1989. 